\title{
determinación del tífulo calcimétrico en los crudos de cemento en functión del valor del módulo hidráulico
}

(«L'Ind ustria Italiana del Cemento», n. ${ }^{\circ} 11$, noviembre 1963)

La conducción económica del horno rotatorio de cemento exige, entre otras cosas, que el valor del módulo hidráulico de la mezcla cruda permanezca constante y comprendido entre límites definidos.

En el proceso por vía seca, por tanto, la preparación de la mezcla se realiza mediante frecuentes controles y correcciones del título calcimétrico del producto de los molinos y homogeneización en los silos.

La determinación del módulo hidráulico—aunque también se realiza por métodos rápidos - resulta excesivamente larga para la premura que requieren las correcciones, y por eso se realiza solamente unas pocas veces al día sobre nuestras medias, como control del conjunto de los resultados calcimétricos obtenidos.

Este control, cuya eficacia tiene, por otra parte, la tara de efectuarse "a posteriori", se hace necesario por la falta de correspondencia entre el título calcimétrico y el módulo hidráulico de mezclas preparadas con materias primas de composición no rigurosamente constante.

El método que se describe muestra como, a base de un cuidadoso estudio de la composición de los materiales de cantera-efectuado de una vez para siempre-, es posible establecer, en la mayoría de los casos, una rigurosa relación entre el título calcimétrico y el módulo hidráulico de una mezcla, mediante la simple determinación de los títulos de las materias primas que lo componen.

\section{principio del procedimiento}

Puesto que el $\mathrm{CaO}$ contenido en una mezcla cruda se encuentra combinado exclusivamente como carbonato, tendremos:

$$
\begin{gathered}
\mathrm{M} . \mathrm{H} .=\frac{\mathrm{CaO}}{\mathrm{SiO}_{2}+\mathrm{Al}_{2} \mathrm{O}_{3}+\mathrm{Fe}_{2} \mathrm{O}_{3}} \\
\mathrm{S=} \mathrm{SiO}_{2}+\mathrm{Al}_{2} \mathrm{O}_{3}+\mathrm{Fe}_{2} \mathrm{O}_{3}+\mathrm{CaO} \text {, en \% } \\
(\mathrm{MgO})_{c}=\mathrm{MgO} \text { presente como } \mathrm{CO}_{3} \mathrm{Mg} \text {, en \% }
\end{gathered}
$$




$$
T_{c}=\frac{C O_{2}}{0,44}, \text { en } \%
$$

De aquí se deduce:

$$
\begin{aligned}
& \text { Puesto que: } \\
& \qquad T_{\mathrm{c}}=\frac{\mathrm{CaO}}{0,56}+1,185 \frac{(\mathrm{MgO})}{0,478} \text {, en } \% \\
& \text { y también: } \\
& T_{\mathrm{c}}=\frac{\mathrm{M} \cdot \mathrm{H} \cdot \mathrm{S}}{0,56(1+\mathrm{M} . \mathrm{H} .)}+1,185 \frac{(\mathrm{MgO})_{c}}{0,478}, \text { en } \%
\end{aligned}
$$

Esta relación permite prever con exactitud qué título calcimétrico, $T_{c}$, debe tener un crudo, conociendo $S$ y $(\mathrm{MgO})_{c}$, para que resulte de un determinado módulo hidráulico, M.H.

$\mathrm{La}$ validez de la fórmula [1] impone como única condición que el $\mathrm{CaO}$ se presente combinado como $\mathrm{CO}_{3} \mathrm{Ca}$; en la práctica, que esté ausente el sulfato cálcico. En caso contrario, los resultados obtenidos con su empleo no son rigurosos, sino solamente aproximados, en una medida tanto menor cuanto más elevado sea el contenido en $\mathrm{SO}_{3}$ de la mezcla.

La presencia en la mezcla de cenizas de pirita-en la cual el $\mathrm{CaO}$ está casi por completo combinado en forma de compuestos distintos del $\mathrm{CO}_{3} \mathrm{Ca}$-se limita, generalmente, a cantidades tan exiguas $(1-3 \%)$ que los errores relativos resultan, sin duda, omisibles.

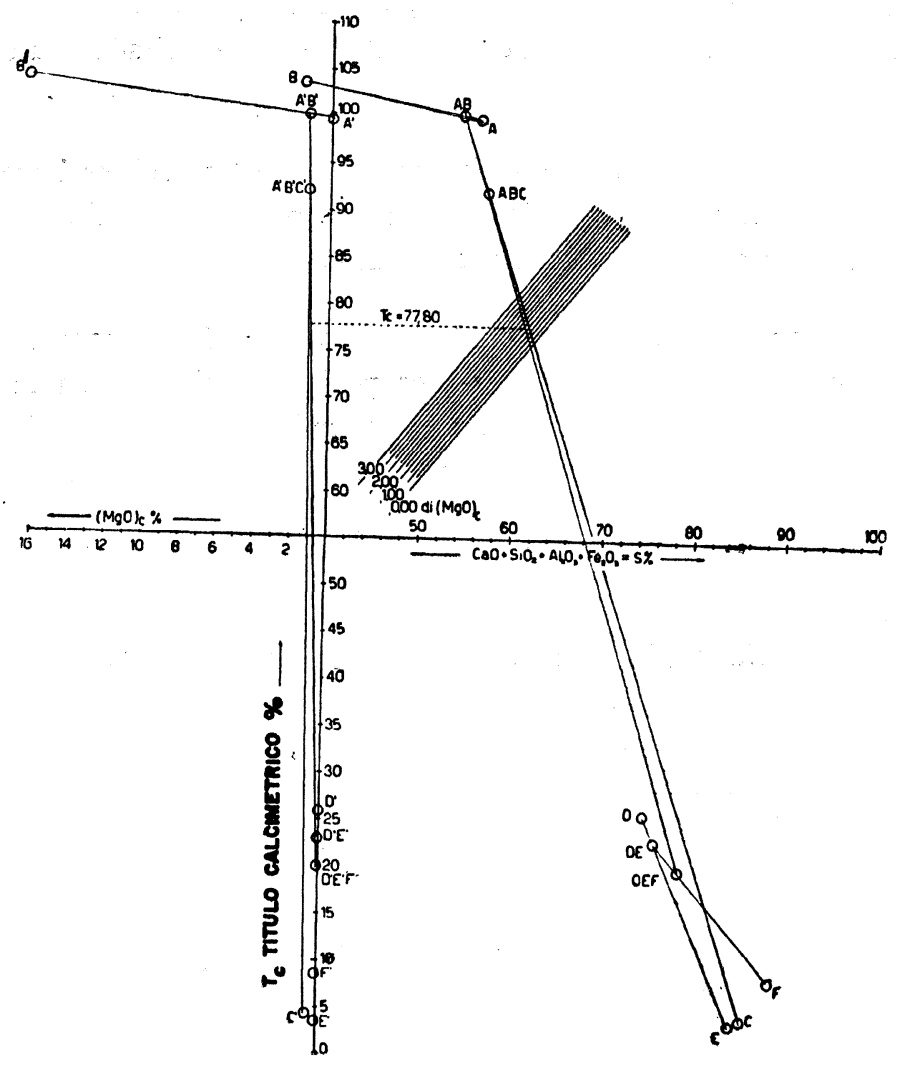




\section{método gráfico para la determinación de Tc en función de $\mathbf{S}$ y de [MgO]c}

La fórmula [1] indica que, fijado el M.H. que convenga, basta conocer los valores de $S$ y de $(\mathrm{MgO})_{c}$ de la mezcla para obtener el título calcimétrico correspondiente.

Los valores $S$ y $(\mathrm{MgO})_{c}$ de la mezcla se pueden determinar fácilmente, en el mismo diagrama que proporciona el valor de $T_{c}$, cuando se conocen los correspondientes valores de las materias primas que componen dicha mezcla.

El diagrama, llamado brevemente de los títulos calcimétricos, viene representado en la figura $1, \mathrm{y}$ consta de una escala de los títulos calcimétricos, que corta ortogonalmente un eje horizontal que contiene los \% de $S$ y de $(M g O)_{c}$. Sobre él se han trazado las rectas $T_{c}=f(S)$ para $M . H .=2,20\left(^{*}\right)$ y contenidos de $(\mathrm{MgO})_{c}$ que varían de 0 a $3 \%$.

El uso correcto del diagrama implica el conocimiento de las composiciones independientes ( $\left.{ }^{* *}\right)$ de los materiales de cantera, las cuales pueden determinarse de una vez para siempre.

Es conveniente que todas las muestras de una cantera o de una materia prima se definan desde el punto de vista químico mediante una o dos únicas composiciones independientes; esto ocurre en la mayoría de los casos prácticos. En otros casos es necesario poder separar físicamente las fases de una misma muestra y someterlo separadamente a la determinación calcimétrica. En el ejemplo de aplicación que se expone más adelante, también se tomará en consideración este caso.

Una vez llevados sobre el diagrama de los títulos calcimétricos los puntos representativos de las composiciones independientes, bien sobre el plano de $S$ o bien sobre el de $(\mathrm{MgO})_{c}$, la determinación calcimétrica de las materias primas en los dosificadores de los molinos, tomada de la mezcla de dos en dos, permite encontrar los valores de $S$ y de $T_{c}$ de la mezcla final, como coordenadas del punto de intersección de la recta de unión de los puntos representativos de las materias primas con la recta del M.H. y del $(\mathrm{MgO})_{c}$ previamente fijados.

La determinación del valor de esta última magnitud se consigue fácilmente mediante un procedimiento análogo realizado sobre el plano $(\mathrm{MgO})_{c}$.

\section{ejemplo numérico de aplicación}

Para la preparación de la mezcla se utiliza caliza, arcilla y material silíceo. La caliza está constituida por carbonato cálcico casi puro (A), mezclado con una especie de caliza dolomítica de composición constante (B) y tierra de cobertura de composición casi constante (C).

(*) Un M.H. $=2,20$ es el más corrientemente usado para los crudos de cemento. Se pueden trazar otros diagramas para diferentes M.H., si fuera preciso, basándose en la ecuación [1].

(**) Entendiéndose por composiciones independientes de una cantera (o materia prima), las composiciones centesimales de algunas muestras elegidas en el menor número posible, pero de manera que la composición de cualquier otra muestra de dicha cantera (o de dicha materia prima) coincida con una de aquéllas, o bien con una mezcla de las mismas. 
La arcilla procede de un barrero de composición constante: se trata de un material plástico que contiene, homogéneamente dispersos, detritus de toba caliza (D). La montera del barrero está constituida por tierra muy magra, casi exenta de carbonatos (E).

El material silíceo tiene una composición constante $(\mathrm{F})$.

Las composiciones independientes de las tres materias primas pueden verse en la tabla 1 . En el diagrama de los títulos calcimétricos de la figura 1 se han señalado sus puntos representativos, bien sobre el plano de $S$ (letras sin acento) o bien sobre el de $(\mathrm{MgO})_{c}$ (letras con acento). Las composiciones independientes $\mathrm{A}, \mathrm{D}, \mathrm{E}, \mathrm{F}$, que no contienen $(\mathrm{MgO})_{c}$, yacen en el diagrama $(\mathrm{MgO})_{c} / T_{\mathrm{c}}$ sobre el eje $T_{c}$.

Para la determinación del título calcimétrico $\left(T_{c}\right)$, en cuyo caso deberá molerse la mezcla para poseer un M.H. $=2,20$, se opera siguiendo el siguiente procedimiento:

- Se determina el título calcimétrico de la arcilla. Se obtiene $23 \%$. Sobre las rectas que unen D y E y $D^{\prime}$ y $E^{\prime}$ se señalan, respectivamente, los dos puntos $D E$ y $D^{\prime} E^{\prime}$, que corresponden con un $23 \%$ de $T_{c}$.

- Se determina el título de la mezcla arcilla-sílice, en proporción determinada en función del módulo silícico a obtener. Resultado: $20 \%$. Se señalan las dos nuevas mezclas ternarias, DEF y $\mathrm{D}^{\prime} \mathrm{E}^{\prime} \mathrm{F}^{\prime}$, sobre la recta de unión, DE-F D'E'-F', para el $20 \%$ de título calcimétrico.

- Sobre una parte alícuota de un testigo de caliza triturada, más o menos sucio de tierra, se elimina con agua la fase terrosa; se deseca con papel de filtro los trocitos de piedra limpia, se tritura y se somete a la determinación calcimétrica. Resultado: 100,20\%. Se señalan los puntos $A B$ y $A^{\prime} B^{\prime}$ para el $100,20 \%$ de $T_{c}$ sobre las rectas de unión de los puntos A y B y A' y B', respectivamente.

- Sobre el resto del testigo de caliza triturada mezclada con tierra, se efectúa también la determinación calcimétrica que suministra el valor del $92 \%$. Sobre la recta de unión, AB-C y $A^{\prime} B^{\prime}-C^{\prime}$, se señalan los dos puntos, $\mathrm{ABC}$ y el $\mathrm{A}^{\prime} \mathrm{B}^{\prime} \mathrm{C}^{\prime}$, para el título del $92 \%$.

- Se enlazan los puntos $A B C$ con $D E F$ y $A^{\prime} B^{\prime} C^{\prime}$ con $D^{\prime} E^{\prime} F^{\prime}$. Se mueve con lentitud la regla, normalmente al eje $T_{c}$, desde el eje $S$ hacia arriba, leyendo los dos valores de $(\mathrm{MgO})_{c}$ que, para las diversas posiciones, marcan la intersección de la regla con la recta $\mathrm{ABC}$-DEF sobre la escala de $M . H .=2,20$ y la intersección de la misma regla con la recta $A^{\prime} B^{\prime} C^{\prime}-D^{\prime} E^{\prime} F^{\prime}$, por proyección, sobre el eje horizontal de $(\mathrm{MgO})_{c}$. Los dos valores coinciden para una determinada ordenada, que representa el $T_{\mathrm{c}}$ buscado. En el ejemplo en cuestión la ordenada $T_{\mathrm{c}}=77,80 \%$.

La construcción geométrica realizada permite, como complemento, efectuar también rápidamente el cálculo de los contenidos de las composiciones independientes que entran a formar parte de la mezcla, haciendo uso de la regla de la palanca sobre los diferentes segmentos trazados. 


\section{T A B L A I}

Composiciones independientes de las materias primas utilizadas en la mezcla

\begin{tabular}{|c|c|c|c|c|c|c|}
\hline & & L I & & & & SILICE \\
\hline & A & B & c & D & $\mathbf{E}$ & $\mathbf{F}$ \\
\hline $\begin{array}{lllllll}\text { P. } & \text { F. } & \ldots & \ldots & \ldots & \ldots & \%\end{array}$ & 43,95 & 46,50 & 11,80 & 18,00 & 9,80 & 6,34 \\
\hline $\begin{array}{lllllll}\mathrm{SiO}_{2} & \ldots & \ldots & \ldots & \ldots & \ldots\end{array}$ & 0,00 & 0,74 & 49,10 & 44,20 & 69,35 & 74,00 \\
\hline $\begin{array}{llllll}\mathrm{Al}_{2} \mathrm{O}_{3} & \ldots & \ldots & \ldots & \ldots & \%\end{array}$ & 0,00 & 1,00 & 19,00 & 12,50 & 0,60 & 7,75 \\
\hline $\begin{array}{llllll}\mathrm{Fe}_{2} \mathrm{O}_{3} & \ldots & \ldots & \ldots & \ldots & \%\end{array}$ & 0,00 & 0,00 & 16,25 & 3,80 & 4,05 & 2,50 \\
\hline $\begin{array}{llllll}\mathrm{Ca} 0 & \ldots & \ldots & \ldots & \ldots & \%\end{array}$ & 55,90 & 35,05 & 1,75 & 14,50 & 2,20 & 4,77 \\
\hline $\begin{array}{llllll}M g 0 & \ldots & \ldots & \ldots & \ldots & \%\end{array}$ & 0,00 & 16,50 & 0,50 & 2,70 & 0,00 & 0,36 \\
\hline $\begin{array}{llllll}\text { Sin } & \operatorname{det} . & \ldots & \ldots & \ldots & \%\end{array}$ & 0,15 & 0,21 & 1,60 & 4,30 & 4,99 & 4,28 \\
\hline Total $\ldots \ldots \ldots$ & 100,00 & 100,00 & 100,00 & 100,00 & 100,00 & 100,00 \\
\hline Mod. Silic. ... ... ... & - & 0,74 & 1,39 & 2,71 & 5,07 & 7,21 \\
\hline Mod. Fund. ... ... & - & - & 1,17 & 3,29 & 2,37 & 3,10 \\
\hline $\mathrm{CO}_{2} \ldots \ldots \ldots \ldots \ldots \%$ & 43,95 & 45,60 & 1,92 & 11,40 & 1,73 & 3,75 \\
\hline $\begin{array}{lllllll}\mathbf{S} & \ldots & \ldots & \ldots & \ldots & \ldots & \%\end{array}$ & 55,90 & 36,79 & 86,10 & 75,00 & 85,21 & 89,02 \\
\hline 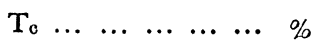 & 99,95 & $.303,60$ & 4,35 & 25,90 & 3,93 & 8,52 \\
\hline 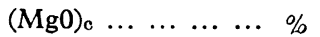 & 0,00 & 16,50 & 0,50 & 0,00 & 0,00 & 0.00 \\
\hline
\end{tabular}

En el caso considerado tenemos:

$$
\begin{aligned}
& \mathbf{A}=68,58 \% \\
& \mathbf{B}=4,91 \% \\
& \mathrm{C}=6,84 \%
\end{aligned}
$$$$
\begin{aligned}
& D=13,53 \% \\
& E=2,07 \% \\
& F=4,07 \%
\end{aligned}
$$

De estos valores, y mediante los datos de la tabla 1, es fácil comprobar la exactitud de los resultados suministrados por el método gráfico confrontados con los teóricos.

\begin{tabular}{ll|c|c|} 
& & Cálculo & $\begin{array}{c}\text { Resultado } \\
\text { gráfico }\end{array}$ \\
\hline M. H. & $\%$ & 2,20 & \\
To & $\%$ & 77,80 & 2,20 \\
$(\mathrm{Mg} 0)_{\mathrm{o}}$ & $\%$ & 0,84 & 77,80 \\
& & & 0,95 \\
\hline
\end{tabular}

Là corrèspondencia entre los resultados es más que satisfactoria. 